\title{
Design and implementation of constrained predictive control simplified algorithm based on particle swarm optimization
}

\author{
Kai-Chen Wang ${ }^{\dagger}$, Ping Ma \\ Dept. of Automation, North China Electric Power University, \\ Baoding, Hebei, 071003, P.R.China \\ E-mail:2669339597@qq.com
}

\begin{abstract}
In order to achieve the purpose of reducing the calculation quantity and improving the computation speed of predictive control, an aggregation algorithm was proposed to design the predictive control simplified algorithm. At the same time, consider the actuators' outputs with restrictions in industrial, the particle swarm optimization was used to design the output constraint on the basis of predictive control simplified algorithm. Finally, the algorithm was applied to boiler denitration control system by PLC in a power plant in Shaanxi Province. The practical result shows that the predictive control algorithm also can be implemented in the device with limited hardware resources and has a good control effect.
\end{abstract}

Keywords: constrained predictive control; Dynamic Matrix Control; particle swarm optimization; algorithm simplification

\section{Introduction}

Dynamic matrix control algorithm is widely used in industrial process. It is a kind of predictive control algorithm which is based on the controlled step response, mainly includes predictive model, roll optimization and feedback correction[1]. The basic thought is that the outputs of controller is according to the principle of internal model control, which smooth the set value and uses the incremental control to achieve the adjustment of zero steady-state error. Finally, realize the prospects predictive control. The algorithm has the advantages of strong robustness and simple prediction model[2].

However, dynamic matrix control algorithm also has its limits, such as complex computation. Therefore, this paper combines the dynamic matrix control algorithm with aggregation algorithm to reduce the computational complexity of dynamic matrix control algorithm. On this basis, use improved particle swarm optimization to design the constraints of controller, which ensure the calculation results have a global optimal solution in every moment. In the end, this algorithm is applied to the project of boiler denitration algorithm 
optimization and automatic control system commissioning in a power plant in Shaanxi province. The practical control results show that the algorithm has fast response speed, strong robustness and the control effect is very well, which has certain engineering application value.

\section{Aggregation Strategy}

Aggregation strategy, one of a simplified algorithm, its core concept is to reduce the dimension or the number of online optimization variables in control horizon and reach the purpose of simplify the computation[3]. In this article, the future optimization variables, which number is $\mathrm{M}$, are mapped to the function of a first-order index form by aggregation strategy. Make the optimization variables with $\mathrm{M}$ dimension are reduced to one dimension matrix and reducing the number of optimization variables during the optimization calculation process, ultimately, reduce the amount of calculation. Assume that the optimization variables for the current moment is $\delta(\mathrm{k})$, The future optimization variables in the form of first-order index is:

$$
\delta(\mathrm{k}+\mathrm{i})=\gamma^{\mathrm{i}} \delta(\mathrm{k}), 0 \leq \mathrm{i} \leq \mathrm{M} .
$$

Among them, $\gamma$ is the aggregation coefficient, which is a preset constant greater than zero. Use Eq.(1) to aggregation can make $\mathrm{M}$ dimensional optimization variables reduce to one dimensional variable, in order to reduce the amount of calculation.

\section{Constrained Dynamic Matrix Simplification Algorithm}

\subsection{Prediction model}

Dynamic matrix control algorithm uses step response of the controlled object that defines as $\{$ ai $\}$ to modeling. Due to the control increment that the dynamic matrix control applies to the actual object is current calculation value, and this value is constantly rolling optimization in every optimization cycle. Therefore, control increment in the future M-1 time doesn't too accurate. At the same time, in order to smooth the changes of control increment and make them change in the same direction in the future M-1 time. So, this paper uses the aggregation function of a first order index form to simplify the $\mathrm{M}$ dimension control increment. According to the Eq.(1), defines $\delta(\mathrm{k})$ as $\Delta u(\mathrm{k})$, the future M-1 time control increment is :

$$
\Delta \mathrm{u}(\mathrm{k}+\mathrm{i})=\gamma^{\mathrm{i}} \Delta \mathrm{u}(\mathrm{k}), 1 \leq \mathrm{i} \leq \mathrm{M}-1 .
$$

In Eq.(2), the future M-1 time control increment can be expressed as a function of $\Delta u(\mathrm{k})$. Therefore, in the rolling optimization process only needs to 
calculate the value of $\Delta u(\mathrm{k})$ and that can predict control increment in the future M-1 time. Use $\Delta \boldsymbol{U}$ to replace $\Delta \boldsymbol{u}_{\mathrm{M}}(\mathrm{k})$ in the predictive model, so new model is :

$$
\widetilde{\mathbf{y}}_{\mathrm{PM}}(\mathrm{k})=\tilde{\mathbf{y}}_{\mathrm{P} 0}(\mathrm{k})+\boldsymbol{A}_{2} \Delta \mathrm{u}(\mathrm{k}) .
$$

$\boldsymbol{A}_{2}$ is $\mathrm{P}$ dimensions column vectors, only need to predetermine the value of $\gamma$ and the model vector $\left\{a_{\mathrm{i}}\right\}, \boldsymbol{A}_{2}$ can be calculated in offline state. The Eq.(3) is new prediction model that is combined with aggregation strategy, its computation is reduced from the original $\mathrm{M} \times \mathrm{P}$ matrix computation to a multiplication of $\mathrm{P} \times 1$ dimensions, which simplifies the calculation process of prediction model.

\subsection{The constraint condition}

In order to meet the actual industrial process actuators constraints limit, $\Delta u(\mathrm{k})$ should satisfy certain constraints in the process of calculation. Make the Eq.(2) and Eq.(3) into the original rolling optimization objective function, and the new objective function is :

$$
\operatorname{minJ}(\mathrm{k})=\left\|\boldsymbol{\omega}_{\mathrm{P}}(\mathrm{k})-\widetilde{\mathbf{y}}_{\mathrm{PM}}(\mathrm{k})\right\|_{\mathrm{Q}}^{2}+\|\Delta \mathbf{U}\|_{\mathrm{R}}^{2} .
$$

The online optimization problem that solves $\Delta u(\mathrm{k})$ in the limited time domain is defined as ${ }^{[4]}$ :

$$
\Delta \mathrm{u}(\mathrm{k})=\arg \min \mathrm{J}(\Delta \mathrm{u}(\mathrm{k})),
$$

Define $\boldsymbol{R}_{\mathrm{i}}$ as $\sum \gamma^{\mathrm{j}}$, and consider the constraint condition of $u(k+i) \in$ $\left[u_{\text {min }}, u_{\text {max }}\right]$, so the first constraint condition of $\Delta u(\mathrm{k})$ is :

$$
\Delta \mathrm{u}_{1}(\mathrm{k}) \in\left[\left[\mathrm{u}_{\min }-\mathrm{u}(\mathrm{k}-1)\right] / \mathrm{R}_{\mathrm{M}-1},\left[\mathrm{u}_{\max }-\mathrm{u}(\mathrm{k}-1)\right] / \mathrm{R}_{\mathrm{M}-1}\right] .
$$

Consider the another constraint condition of $\Delta u(\mathrm{k}+\mathrm{i}) \in\left[\Delta u_{\min }, \Delta u_{\max }\right]$, that can get the second constraint condition of $\Delta u(\mathrm{k})$ :

$$
\Delta \mathrm{u}_{2}(\mathrm{k}) \in\left\{\begin{array}{l}
{\left[\Delta u_{\min } / \gamma^{M-1}, \Delta u_{\max } / \gamma^{M-1}\right], \gamma>1} \\
{\left[\Delta \mathrm{u}_{\min }, \Delta \mathrm{u}_{\max }\right]}
\end{array}\right]
$$

Consider the above of two constraint conditions, the final constraint range of $\Delta u(\mathrm{k})$ is :

$$
\Delta \mathrm{u}(\mathrm{k}) \in\left\{\begin{array}{l}
{\left[\max \left(\Delta \mathrm{u}_{\min }, \frac{\mathrm{u}_{\min }-\mathrm{u}(\mathrm{k}-1)}{\mathrm{R}_{\mathrm{M}-1}}\right), \min \left(\Delta \mathrm{u}_{\max }, \frac{\mathrm{u}_{\max }-\mathrm{u}(\mathrm{k}-1)}{\mathrm{R}_{\mathrm{M}-1}}\right)\right], \gamma>1} \\
{\left[\max \left(\frac{\Delta \mathrm{u}_{\min }}{\gamma^{\mathrm{M}-1}}, \frac{\mathrm{u}_{\min }-\mathrm{u}(\mathrm{k}-1)}{\mathrm{R}_{\mathrm{M}-1}}\right), \min \left(\frac{\Delta \mathrm{u}_{\max }}{\gamma^{\mathrm{M}-1}}, \frac{\mathrm{u}_{\max }-\mathrm{u}(\mathrm{k}-1)}{\mathrm{R}_{\mathrm{M}-1}}\right)\right], \gamma<1}
\end{array} .\right.
$$

\subsection{Optimization calculation based on PSO}


Solve $\Delta u(\mathrm{k})$ in a limited range can also be regarded as a optimization problem of $\Delta u(\mathrm{k})$ in a limited range $\mathrm{e}^{[5]}$. Therefore, this article uses particle swarm optimization algorithm, take the Eq.(4) as the optimization objective function and the Eq.(5), Eq.(8) as the constraint conditions, to optimize $\Delta u(\mathrm{k})$ online in a finite one-dimensional interval.

Select the value of $\gamma$ and choose the Eq.(8) as the initial constraint condition of the particle swarm optimization algorithm. The basic calculation process of $\Delta u(\mathrm{i})$ is :

(1) Initial the value of $u(\mathrm{i}), \Delta u(\mathrm{i})$ and the particle population,

(2) Calculate the optimization index of each particle according to the optimization objective function,

(3) For each particle, compare the current optimization index with its historical best optimization index, if the current optimization is better, update the optimal location of a single particle history,

(4) For each particle, compare the historical optimal index with the historical optimal index of the population, if better and then update the optimal location of particle history within the population,

(5) Update the value of $u(\mathrm{i}), \Delta u(\mathrm{i})$ and $\boldsymbol{\omega}$,

(6) To determine whether the iterative process achieves the maximum number of iterations, if achieves and then outputs the value of $u(\mathrm{i})$ and $\Delta u(\mathrm{i})$. if not, return to the step of (3) and continue the iterations.

\section{The Control Effect in Power Plant}

\subsection{Control scheme of SCR denitrification based on predictive control}

The predictive control simplified algorithm has been applied into the project of redesign the denitrification system in a power plant in Shaanxi Province. This project uses SIEMENS S7-200 PLC to realize the predictive control algorithm. In order to achieve the purpose of data acquisition and the transmission of control quantity, the PLC communicates with communication card of DCS, which is called FBM224, by MODBUS485 communication protocol of industrial standard. Simultaneously, retain the original control scheme of denitrification in DCS. When PLC operation failure, it can switch to the original scheme in time and without interference and ensure the safe and stable operation of the denitration system.

The automatic control strategy for the original denitrification of the power plant is an open loop control, and same problems are discovered during the operation:

(1) When the load is stable, spray ammonia at the inlet of the SCR reactor is high, which leads to the crystallization of the air preheater is serious, 
(2) When the load goes up or down, the NOx emissions is easy to exceed,

(3) When the load is stable and the efficiency of denitrification is constant, the NOx emission of SCR is quite different under the different stable load, which forces the operator to adjust the denitrification efficiency frequently according to the working conditions.

In view of such problems, combined with the predictive control algorithm, an optimal algorithm based on feedforward predictive control is proposed in this paper. Combining predictive control technology in feedforward, which can quickly and accurately estimate the amount of ammonia required in the SCR reactor. Ensure that NOx emissions in flue gas can reach the national standard while reducing the amount of excess ammonia injection. The Fig.1 is the principle diagram of the optimization algorithm.

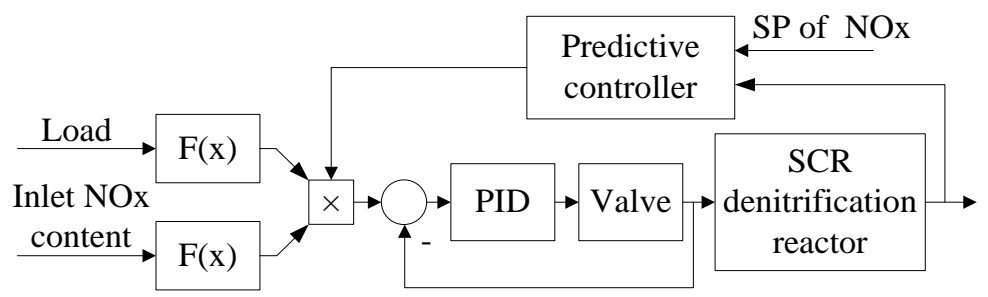

Fig.1. The principle diagram of the optimization algorithm

\subsection{The control results}

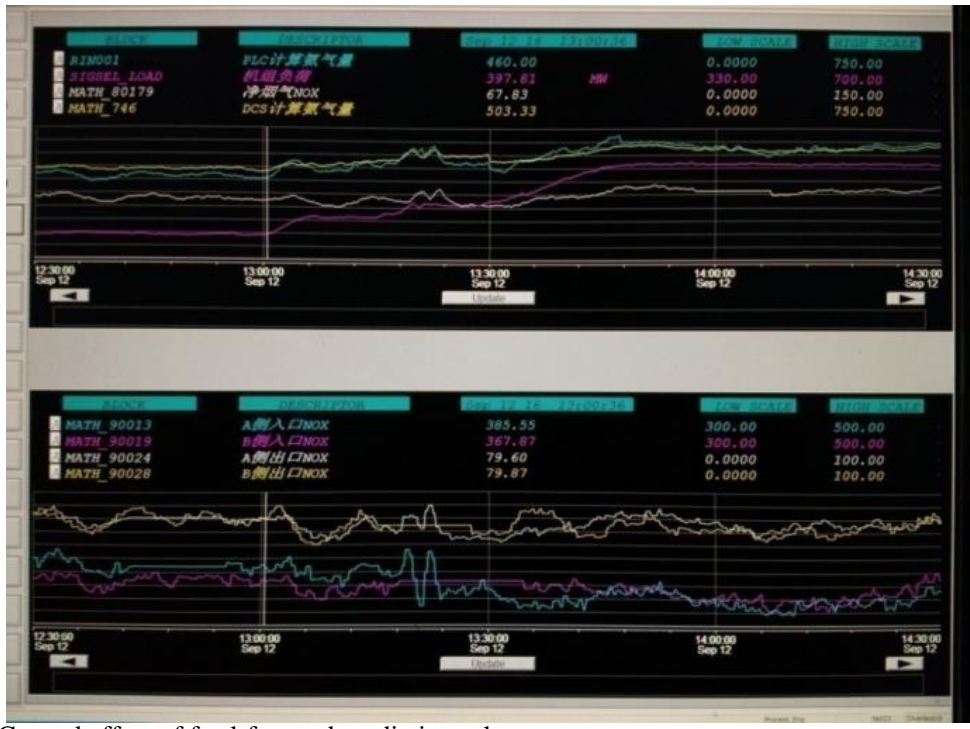

Fig.2. Control effect of feed-forward prediction scheme 
The Fig. 2 is the Control effect of feed-forward prediction scheme. When the load is stable, compared with the original scheme, the ammonia calculation of feedforward predictive control algorithm is about $40 \mathrm{mg} / \mathrm{Nm}^{3}$ lower, which is conducive to reducing the amount of ammonia escape. When the load is increased from $400 \mathrm{MW}$ to $600 \mathrm{MW}$, the ammonia calculation of the prediction scheme can be increased in advance and in a rapid manner, ensure that NOx emissions of the SCR outlet are not exceeded. Between 13:15 to 13:30, the NOx content of SCR inlet had a greater perturbation, the prediction scheme could respond to the perturbation in time and effectively suppress the fluctuation of NOx emission. In the whole process of the load increasing, the NOx emissions can be basically maintained between $70 \sim 80 \mathrm{mg} / \mathrm{Nm}^{3}$.

\section{Conclusion}

A dynamic matrix predictive control simplification algorithm is proposed in this paper. Compared to original dynamic matrix control, the new control increment decreases from $\mathrm{M}$ dimensions to one dimension, which reduces the computation and improves the efficiency. Meanwhile, due to the computation of the control increment is a one-dimensional function, this work proposes to use the particle swarm optimization to calculate the control increment in the finite time domain with constraint conditions. That not only avoid the complex matrix calculation, but also avoid the results of the calculation without the global optimal solution. This algorithm has been realized in a power plant in Shaanxi Province. The practical results show that the controller has fast response speed, stable control quantity, strong robustness and its control effect is satisfactory.

\section{Reference}

1. Y.G. Xi, Predictive Control, 2nd edn. (National Defence Industry Press, Beijing, 2013).

2. Y.F. Liu, G. Wu, H.H. Wei, etc, Stair-like dynamic matrix control and its application in temperature control system. Journal of University of Science and Technology of China, Vol.32(3), pp.92-97, 2002.

3. N.N. Zhu, Analysis of model predictive control simplified algorithm, Master's thesis, Zhejiang University (2011).

4. R. Bao, D.F. He, K.H. Zheng, Stair-like constrained predictive control based on golden section method. Journal of Shanghai Jiaotong University, Vol.46(12), pp.1940-1944, 2012.

5. Y. Wu, Application of generalized predictive control based on improved particle swarm optimization in the water-level system of boilers, Master's thesis, Taiyuan University of Technology (2013). 